\title{
Transcriptome and proteome quantification of a tumor model provides novel insights into post-transcriptional gene regulation
}

\author{
Christoph Jüschke ${ }^{1}$, Ilse Dohnal ${ }^{2}$, Peter Pichler ${ }^{2,3}$, Heike Harzer ${ }^{1}$, Remco Swart ${ }^{4}$, Gustav Ammerer ${ }^{2}$, \\ Karl Mechtler ${ }^{1,3}$ and Juergen A Knoblich ${ }^{1 *}$
}

\begin{abstract}
Background: Genome-wide transcriptome analyses have given systems-level insights into gene regulatory networks. Due to the limited depth of quantitative proteomics, however, our understanding of post-transcriptional gene regulation and its effects on protein-complex stoichiometry are lagging behind.

Results: Here, we employ deep sequencing and the isobaric tag for relative and absolute quantification (iTRAQ) technology to determine transcript and protein expression changes of a Drosophila brain tumor model at near genome-wide resolution. In total, we quantify more than 6,200 tissue-specific proteins, corresponding to about 70\% of all transcribed protein-coding genes. Using our integrated data set, we demonstrate that post-transcriptional gene regulation varies considerably with biological function and is surprisingly high for genes regulating transcription. We combine our quantitative data with protein-protein interaction data and show that post-transcriptional mechanisms significantly enhance co-regulation of protein-complex subunits beyond transcriptional co-regulation. Interestingly, our results suggest that only about $11 \%$ of the annotated Drosophila protein complexes are co-regulated in the brain. Finally, we refine the composition of some of these core protein complexes by analyzing the co-regulation of potential subunits.

Conclusions: Our comprehensive transcriptome and proteome data provide a valuable resource for quantitative biology and offer novel insights into understanding post-transcriptional gene regulation in a tumor model.
\end{abstract}

\section{Background}

Eukaryotic gene expression involves transcription, mRNA processing and decay, translation, and protein modification and degradation. Each of these steps is tightly regulated to ensure the proper function and stability of the biological system [1]. While genome and transcriptome data have accumulated rapidly since the advent of microarray and deep-sequencing technologies, the limited depth of quantitative proteomics has inhibited similar progress in post-transcriptional gene regulation. Therefore, transcript levels are still routinely used as the only measure for gene expression in high-throughput approaches. Several studies, however, have reported a low

\footnotetext{
*Correspondence: juergen.knoblich@imba.oeaw.ac.at

1 Institute of Molecular Biotechnology of the Austrian Academy of Sciences (IMBA), Dr Bohr-Gasse 3, 1030 Vienna, Austria

Full list of author information is available at the end of the article
}

correlation between transcript and protein levels [2-6], highlighting the importance of post-transcriptional processes as well as the limited predictive value of transcripts for protein expression. Hence, a better understanding of genetic information processing requires consideration of quantitative information at every step of gene expression control.

Recently, studies have begun to address this problem systematically by acquiring large-scale quantitative mRNA and protein data from bacteria $[7,8]$, yeasts [9-11] and cell lines $[12,13]$. For complex tissues of higher organisms, however, such information is still rare. Quantitative analyses are either restricted to a few hundred genes due to limited proteome coverage $[5,14]$ or they focus on cultured cell lines that might have lost properties of their tissue of origin over time [12,13,15-17].

We therefore set out to address this problem using a complex neural tissue in wild-type state and tumor state. 
The Drosophila brain arises from neural stem cells called neuroblasts that undergo repeated rounds of asymmetric cell division giving rise to self-renewing neuroblasts and terminally differentiating neurons [18-20]. In homozygous brain tumor (brat) mutants, some neuroblast divisions become symmetric leading to the formation of excess neuroblasts at the expense of neurons. This causes an uncontrolled expansion of the neuroblast pool and results in the formation of a large brain tumor [21-23]. These tumors can be transplanted into host flies, where they become aneuploid and undergo metastasis [24]. Normally, tumor formation is lethal during larval development, but hypomorphic mutants can survive until adulthood, and the flies harbor large proliferating neuroblast tumors in their brains. The simple cytology of the developing Drosophila brain and the reproducibility of tumor formation have made brat mutants a well-studied example for stem-cellderived tumor formation.

Here, we performed an in-depth integrative analysis of transcript and protein expression data from a complex metazoan tissue, comparing Drosophila brain tumor (brat) versus wild-type heads. Using relative protein quantification with mass spectrometry (isobaric tag for relative and absolute quantification (iTRAQ)) [25], we determined relative expression levels for more than 6,200 proteins, corresponding to about $70 \%$ of all transcribed protein coding genes.

By investigating transcript-protein correlations, namely the change of correlation between the normal and tumorous state, we identify biological processes that are strongly regulated by post-transcriptional mechanisms. Furthermore, we demonstrate that the stoichiometric expression of protein-complex subunits is controlled by a two-tiered mechanism involving co-expression on the mRNA level followed by post-transcriptional fine-tuning. Surprisingly, our data suggest that co-regulation of protein-complex subunits is the exception and not the rule. Finally, our comprehensive data set provides a valuable resource for quantitative systems-level analyses.

\section{Results and discussion}

\section{About $60 \%$ of protein-coding transcripts are expressed in wild-type and brat fly heads}

To obtain sufficient amounts of material for transcriptome and proteome analyses we established a workflow to collect large numbers of homozygous brat mutant fly heads (Figure 1A). Homozygous mutant female flies exhibited a tumor penetrance of $100 \%$, and the median adult survival time was reduced to 10 days (Figure 1B).

For transcriptome analysis, total RNA samples from brat and wild-type female fly heads were prepared in biological triplicates, analyzed by strand-specific paired-end mRNA sequencing and quantified by mapping the reads to the Drosophila genome. The average expression levels (measured as fragments per kilobase of transcript per million mapped fragments (FPKM)) showed a bimodal distribution with most genes following a normal distribution centered at approximately 12 FPKM, and a minority forming a 'shoulder' to the left of the distribution (Figure 2A). Transcripts in the left shoulder with FPKM $<1$ were shown to occur in less than one copy per cell and to have functions not typical for the cell type [26]. Hence, we excluded these very low abundant, presumably non-functional, transcripts from our analysis.

We found that transcript expression correlated well between wild-type and brat samples (Pearson correlation coefficient $r=0.85$, Figure 2B) indicating that the tumors maintain many characteristics of the corresponding wild-type tissue. In total, we were able to quantify transcripts from 8,333 of the 13,781 annotated protein-coding Drosophila genes in both wild-type and brat mutant heads. On average $82 \%$ of all annotated transcripts were expressed in adult female fly heads for each second-level Kyoto Encyclopedia of Genes and Genomes (KEGG) category [27] (Figure 2C). The highest absolute mRNA expression levels were found in the categories 'Energy Metabolism' and 'Translation', which agrees well with recent data from fission yeast [11], and in the category 'Sensory System', consistent with the specific functions of the analyzed tissue (Figure 2D).

\section{Quantification of approximately $70 \%$ of the brain tumor proteome}

In a pilot proteomic study, brat and wild-type fly head samples were labeled in duplicate with 4-plex iTRAQ [25], separated by two-dimensional liquid chromatography and measured by online tandem mass spectrometry. We quantified expression changes of 68,391 peptides with 8,017 unique sequences, corresponding to 1,311 unique proteins at a protein false discovery rate (FDR) of $5 \%$. We refer to this first data set as iTRAQ \#1.

Taking the number of quantified protein-coding transcripts as an estimate for the total number of expressed proteins, we set out to increase the proteome coverage of our iTRAQ analysis. For this, we optimized the proteomics workflow by employing digestion with two proteases, high-resolution two-dimensional chromatography with extensive fractionation, combined collisional-induced dissociation (CID)/higher energy C-trap dissociation (HCD) and electron transfer dissociation (ETD)/HCD fragmentation [28], and multiple search engines using Protein Discoverer (Thermo Fisher Scientific). In all further analyses and discussion we refer to this as the optimized iTRAQ data set (iTRAQ \#2).

Each sample was digested separately with two specific proteolytic enzymes, trypsin and LysC, and 

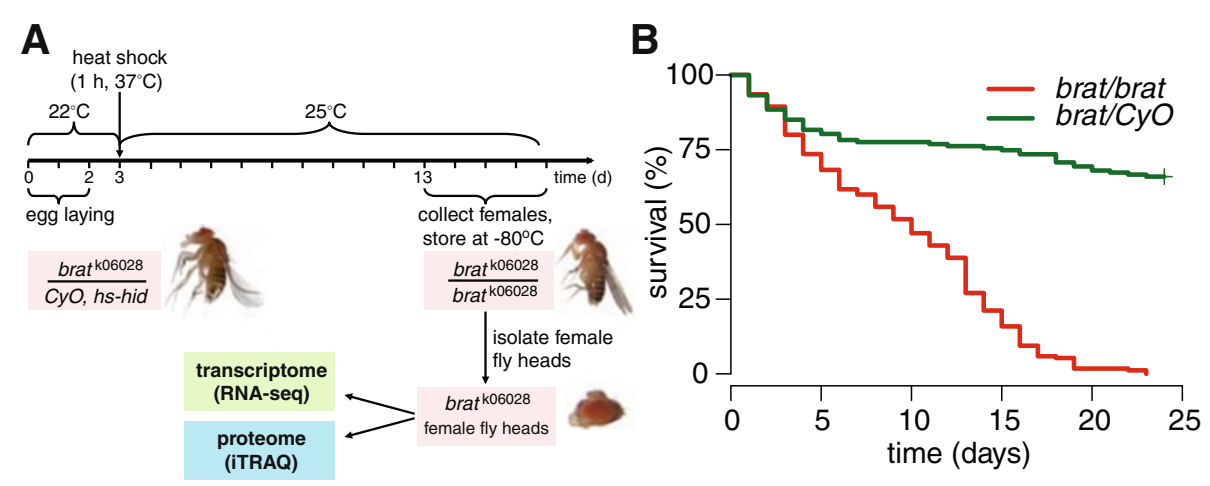

Figure 1 Sample preparation workflow. (A) Breeding scheme for generation of homozygous mutant fly heads. Fly eggs were collected over 2 days. On the third day, heterozygous offspring were killed by heat shock. Adult female flies were collected 1 to 3 days after pupal eclosion and snap-frozen and their heads were isolated. (B) Kaplan-Meier survival plot of homozygous brat mutant (red) versus control flies (green). The tumor penetrance is $100 \%$ reducing the median adult survival time to 10 days. Data were pooled from three independent experiments with 170 brat and 163 control adult female flies in total.

labeled in duplicate with 4-plex iTRAQ. Trypsinand LysC-digested samples were fractionated by high-resolution strong cation exchange (SCX) chromatography with a two-dimensional gradient into 85 and 118 fractions, respectively, and then analyzed by liquid chromatography-tandem mass spectrometry (LCMS/MS) on a LTQ-Orbitrap Velos (Figure 3A). Protein quantification of technical iTRAQ replicates correlated very well $(r=0.99$, Additional file 1: Figure S1A).

To confirm the iTRAQ data, we quantified 34 proteins by selected reaction monitoring (SRM) [29], an alternative label-free protein quantification method. We observed a high technical and biological reproducibility of iTRAQ and SRM protein quantification $(r=0.83$, Additional file 1: Figure S1B and $r=0.73$, Additional file 1: Figure S1C, respectively). In addition, a comparison of iTRAQ and SRM measurements showed that the level of regulation appeared higher for SRM than for iTRAQ. This is in agreement with previous observations reporting an underestimation of protein expression changes ('ratio compression') for iTRAQ [30,31]. To account for this effect, we performed correlation analyses between the different data sets using rank correlations.

In total, we were able to determine the relative expression of 278,763 peptides (FDR < 1.6\%) containing 65,742 unique sequences, with $166,615(60 \%)$ of the peptides from trypsin-digested and 112,148 (40\%) from LysCdigested samples. The trypsin and LysC samples were largely complementary in their contribution of unique peptides for quantification (Additional file 1: Figure S1D).

The peptide sequences mapped unambiguously to 6,277 FlyBase-annotated protein-coding genes at a protein FDR of $5 \%$. The FDR of the integrated data set was lower since we combined the proteome with transcriptome data and performed correlation analyses only for genes with both quantitative protein and transcript data available. Expression changes for $75 \%$ of the proteins were determined in both trypsin- and LysC-digested samples (Additional file 1: Figure S1E), and showed good reproducibility $(r=$ 0.7 , Additional file 1: Figure S1F). We found that $18 \%$ of the proteins were exclusively quantified in the trypsin sample and $7 \%$ in the LysC sample.

For $93 \%(5,840$ of 6,277$)$ of all quantified proteins we were able to quantify the corresponding transcripts as well, and the correlation between mRNA and protein expression changes was very similar to a previous study in cell lines (Spearman's rank correlation $\rho=0.61$ versus $\rho=0.58$ to 0.63 in [12]). Considering that 8,333 proteincoding genes were expressed in the samples according to transcriptome analysis, we have quantified 70\% (5,840 of $8,333)$ of all expressed proteins. Therefore, our data represent one of the most complete quantitative proteomics analyses of a complex tissue comparing two physiological states.

\section{High quantitative proteome coverage for Kyoto Encyclopedia of Genes and Genomes pathways and abundant transcripts}

To further evaluate the quality of our data, we correlated proteome coverage with expression levels, physicochemical properties and annotated functions. Using the codon adaptation index as a predictor for expression levels [32], we found increased coverage for proteins predicted to be more abundant (Additional file 2: Figure S2A). Furthermore, we observed increased coverage for proteins encoded by more abundant transcripts. For mRNAs with FPKM $>10$, for example, we obtained a protein coverage of more than $82 \%$ (Figure 3B). As shown previously [33], we detected a higher proteome coverage for larger proteins since they generally produce a larger number 


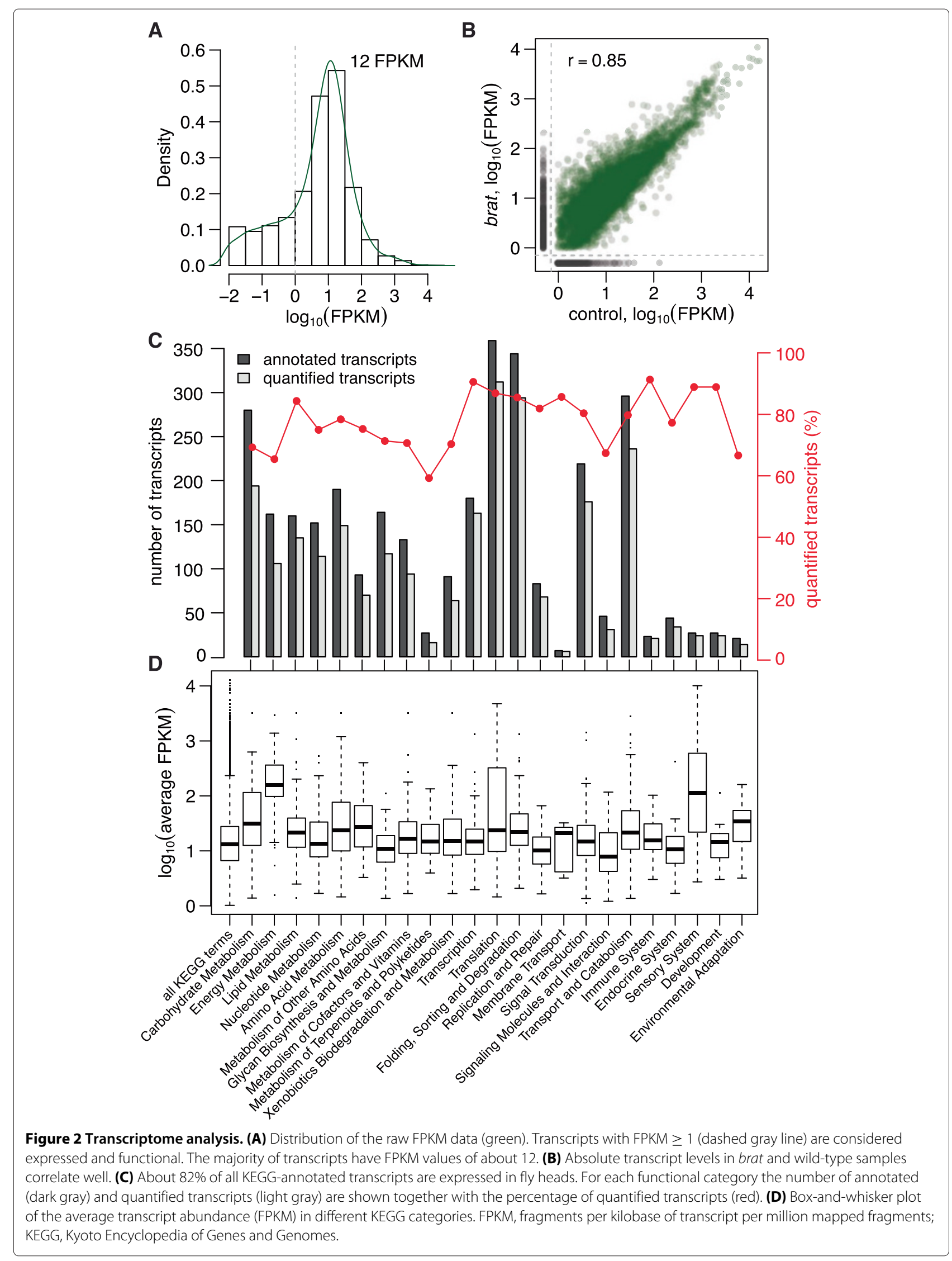




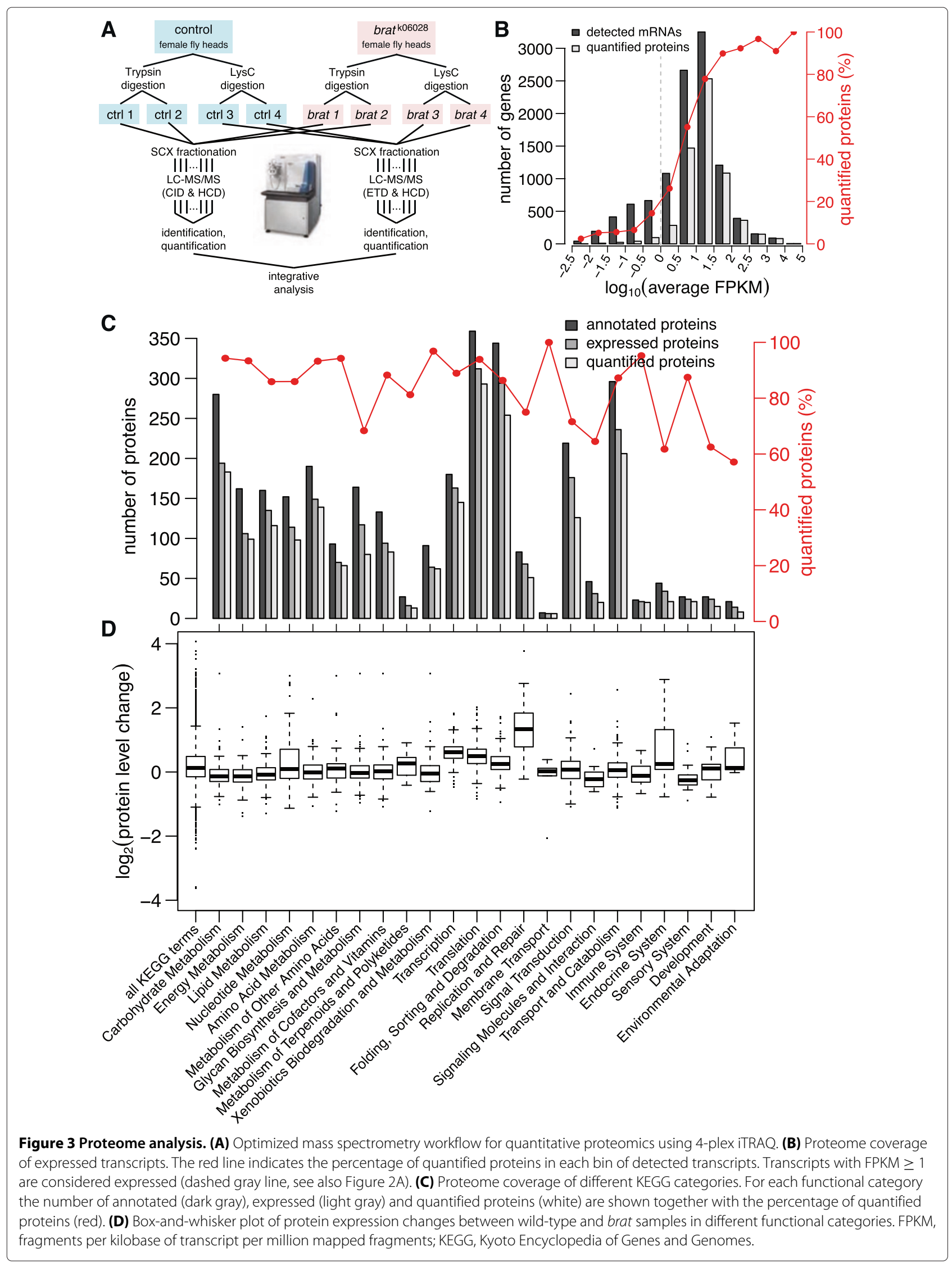


of different peptides upon digestion (Additional file 2: Figure S2B). Only extremely hydrophobic proteins were under-represented and covered by less than $50 \%$, presumably due to reduced solubility during sample preparation (Additional file 2: Figure S2C), whereas protein coverage was higher than $60 \%$ for the full range of isoelectric points (Additional file 2: Figure S2D). Most importantly, our analysis quantified on average $86 \%$ of the expressed proteins within each second-level KEGG pathway category with only five categories covered by less than $70 \%$ (Figure 3C). Thus, all biological pathways are well represented and our data are a good representation of the entire expressed proteome.

\section{DNA replication and damage repair pathways are upregulated in tumors}

To identify deregulated biological processes in the tumors, we performed functional pathway enrichment analyses for transcript and protein level changes using a $z$-value cut-off of 2. The set of pathways over-represented in upregulated transcripts and proteins largely overlapped (KEGGterm enrichment analysis of upregulated transcripts and proteins: Tables 1 and 2, respectively). As expected for proliferating tumor tissue, the KEGG pathway 'DNA replication' was strongly enriched among proteins upregulated in brat (Table 2). Surprisingly, however, this list also contained multiple terms associated with DNA damage repair like 'Mismatch repair', 'Nucleotide excision repair' and 'Base excision repair' (Table 2), and proteins in the KEGG category 'Replication and repair' were most highly upregulated in the tumors (Figure 3D). Although genomic instability does not cause brain tumor formation in Drosophila [34], our results suggest that the DNA damage reported for brat tumors upon transplantation [24] might already be present in the primary tumor.

\section{Hydrophobic proteins tend to be downregulated in the tumors}

Our comprehensive data set of protein expression changes between wild-type and brat samples allowed us to test for correlations with specific primary sequence features (Figure 4). Protein expression changes neither correlated with molecular weight $(\rho=0.02)$ nor isoelectric point ( $\rho=0$, data not shown). However, hydrophobic proteins were preferentially downregulated in the tumor ( $\rho=$ $-0.25, P=1.3 \times 10^{-89}$, Figure $4 \mathrm{~A}$ ). A possible explanation is that brat mutant neuroblasts fail to differentiate into neurons and hence do not upregulate the multitude of transmembrane proteins required for mature neuronal function $[23,35,36]$.

Table 1 Kyoto Encyclopedia of Genes and Genomes based enrichment analysis of upregulated transcripts

\begin{tabular}{|c|c|c|c|}
\hline $\begin{array}{l}\text { Kyoto Encyclopedia of Genes } \\
\text { and Genomes pathway }\end{array}$ & Enrichment & $P$ value & $\begin{array}{l}\text { Adjusted } \\
P \text { value }\end{array}$ \\
\hline Replication and repair & 18.4 & $1.3 \times 10^{-32}$ & $2.05 \times 10^{-30}$ \\
\hline Genetic information processing & 4.19 & $1.92 \times 10^{-29}$ & $1.52 \times 10^{-27}$ \\
\hline DNA replication & 21 & $6.03 \times 10^{-17}$ & $3.18 \times 10^{-15}$ \\
\hline Nucleotide excision repair & 15.5 & $6.69 \times 10^{-12}$ & $2.64 \times 10^{-10}$ \\
\hline Mismatch repair & 22.6 & $5.33 \times 10^{-11}$ & $1.68 \times 10^{-9}$ \\
\hline RNA transport & 6.87 & $1.16 \times 10^{-10}$ & $3.05 \times 10^{-9}$ \\
\hline Transcription & 5.3 & $1.21 \times 10^{-9}$ & $2.72 \times 10^{-8}$ \\
\hline Translation & 3.59 & $9.41 \times 10^{-9}$ & $1.86 \times 10^{-7}$ \\
\hline Pyrimidine metabolism & 6.56 & $8.27 \times 10^{-7}$ & $1.45 \times 10^{-5}$ \\
\hline Basal transcription factors & 10 & $9.61 \times 10^{-7}$ & $1.52 \times 10^{-5}$ \\
\hline Non-homologous end-joining & 31.8 & $2.75 \times 10^{-6}$ & $3.95 \times 10^{-5}$ \\
\hline Homologous recombination & 13.2 & $2.68 \times 10^{-5}$ & 0.000353 \\
\hline Base excision repair & 11.9 & $4.69 \times 10^{-5}$ & 0.00057 \\
\hline Ribosome biogenesis in eukaryotes & 5.05 & $7.07 \times 10^{-5}$ & 0.000798 \\
\hline Spliceosome & 4.18 & 0.00014 & 0.00148 \\
\hline Progesteron-mediated oocyte maturation & 6.5 & 0.000292 & 0.00271 \\
\hline Endocrine system & 6.5 & 0.000292 & 0.00271 \\
\hline Nucleotide metabolism & 3.45 & 0.000361 & 0.00317 \\
\hline Organismal systems & 3.33 & 0.00158 & 0.0131 \\
\hline
\end{tabular}


Table 2 Kyoto Encyclopedia of Genes and Genomes based enrichment analysis of upregulated proteins

\begin{tabular}{lccc}
\hline $\begin{array}{l}\text { Kyoto Encyclopedia of Genes } \\
\text { and Genomes pathway }\end{array}$ & Enrichment & $\boldsymbol{P}$ value & $\begin{array}{c}\text { Adjusted } \\
\boldsymbol{P} \text { value }\end{array}$ \\
\hline Replication and repair & 19.5 & $6.32 \times 10^{-26}$ & $9.99 \times 10^{-24}$ \\
DNA replication & 34.3 & $2.17 \times 10^{-24}$ & $1.72 \times 10^{-22}$ \\
Nucleotide excision repair & 19.2 & $5.58 \times 10^{-12}$ & $2.94 \times 10^{-10}$ \\
Homologous recombination & 28.8 & $1.09 \times 10^{-10}$ & $4.32 \times 10^{-9}$ \\
Mismatch repair & 27.2 & $1.87 \times 10^{-10}$ & $5.89 \times 10^{-9}$ \\
Base excision repair & 22.6 & $1.25 \times 10^{-8}$ & $3.29 \times 10^{-7}$ \\
Genetic information processing & 2.56 & $1.63 \times 10^{-7}$ & $3.69 \times 10^{-6}$ \\
Pyrimidine metabolism & 8.09 & $4.04 \times 10^{-7}$ & $7.98 \times 10^{-6}$ \\
Non-homologous end-joining & 32.3 & $7.04 \times 10^{-5}$ & 0.00124 \\
Nucleotide metabolism & 4.26 & 0.000125 & 0.00198 \\
Progesterone-mediated oocyte maturation & 7.35 & 0.00056 & 0.00737 \\
Endocrine system & 7.35 & 0.00056 & 0.00737 \\
Purine metabolism & 3.54 & 0.00373 & 0.0453 \\
\hline
\end{tabular}

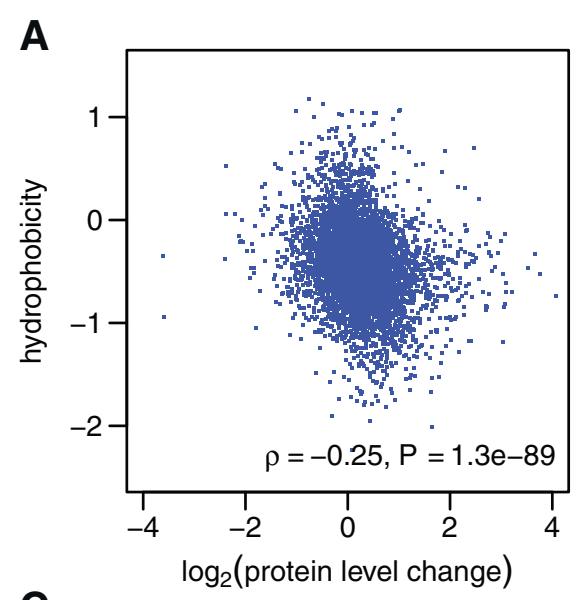

C

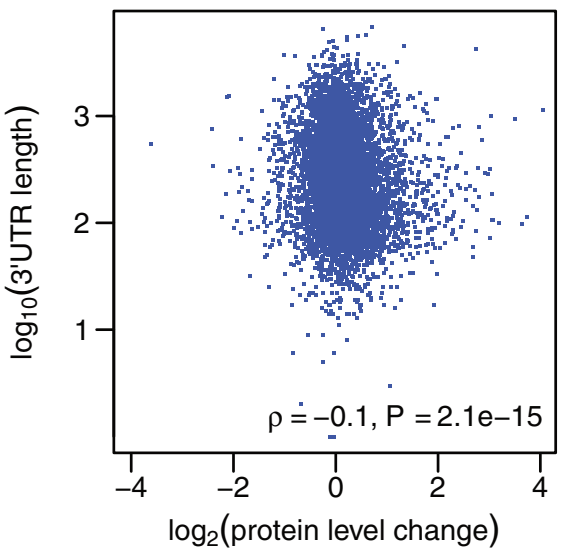

B

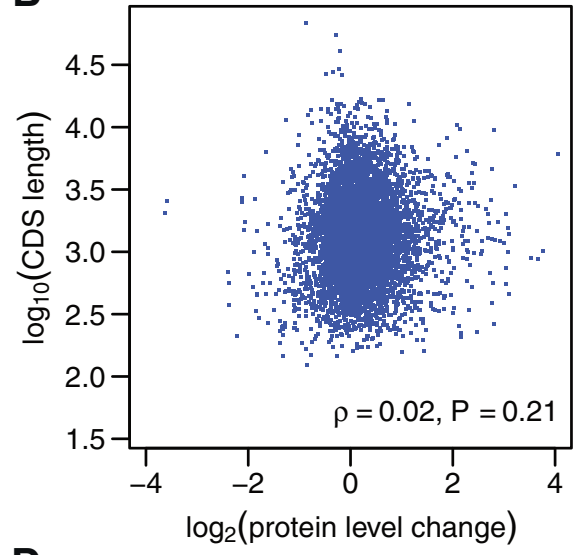

D

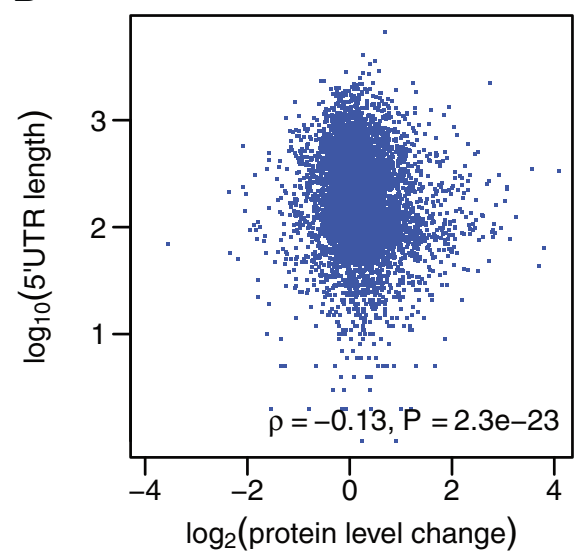

Figure 4 Correlation of sequence features with changes in protein expression. (A) Protein hydrophobicity negatively correlates with protein level change. (B) Length of the coding sequence does not affect protein expression changes. (C) Lengths of the $3^{\prime}$ and (D) $5^{\prime}$ UTRs negatively correlate with protein upregulation. CDS, coding sequence; UTR, untranslated region. 
Shorter 3' and 5' UTRs correlate with protein upregulation While protein level changes did not correlate with the length of coding sequences ( $\rho=0.02$, Figure 4B), we detected a very low but significant negative correlation with the length of the 3 ' and 5' untranslated regions (3' UTR: $\rho=-0.1, P=2.1 \times 10^{-15}$, Figure 4C; 5' UTR: $\rho=$ $-0.13, P=2.3 \times 10^{-23}$, Figure 4D). To determine if upand downregulated proteins were differentially affected by UTR length, we divided the pool of quantified proteins into two subsets: downregulated and upregulated relative to the median, and analyzed the correlation for these subsets separately. Interestingly, only upregulated proteins with shorter UTR length were more highly upregulated (3' UTR: $\rho=-0.12, P=1.8 \times 10^{-10}$; 5 UTR: $\rho=-0.11$, $\left.P=3.5 \times 10^{-9}\right)$, whereas downregulated proteins did not have a significant correlation with UTR length. On average, transcripts with shorter UTRs are expected to have less binding sites for regulatory factors like miRNAs and RNA-binding proteins, and thus, are less susceptible to post-transcriptional control. Our observation is consistent with data showing that 3' UTR shortening increases mRNA stability and protein expression, and leads to oncogene activation in cancer cells [37,38]. Interestingly, our data suggest that this effect might occur not only for 3' UTRs but also for 5' UTRs.

\section{Genes involved in transcription are strongly regulated by post-transcriptional control}

Next, we considered whether mRNA abundance had an effect on the expression change of proteins in the tumor. Interestingly, we observed a negative correlation of wild-type transcript abundance with protein level change $\left(\rho=-0.29, P=2.5 \times 10^{-118}\right.$, Additional file 3: Figure S3A), whereas the opposite was not the case: transcript levels in the tumor did not correlate with protein level change (Additional file 3: Figure S3B). Our results indicate that proteins encoded by transcripts, which are lowly expressed in wild-type samples, have an increased propensity for being upregulated in the tumors.

Quantification of both mRNA and protein level changes allowed us to test the contribution of post-transcriptional mechanisms to the proteome alterations that occur in brat brain tumors. For this, we compared how well changes in protein levels correlate with changes in the corresponding transcripts. Overall protein and mRNA changes correlated similarly to a previous analysis in human cell lines ( $\rho=0.61$ versus $\rho=0.58$ to 0.63 in [12]). Surprisingly, when investigating the different biological pathways individually, we found that the correlations were highly variable. We used random sampling to control for the different number of genes in each KEGG category to identify categories that deviate significantly from the global correlation (Table 3 and Figure 5).

\section{Table 3 Biological pathways exhibiting significant alterations in post-transcriptional regulation}

\begin{tabular}{lcc}
\hline $\begin{array}{l}\text { Kyoto Encyclopedia of } \\
\text { Genes and Genomes pathway }\end{array}$ & Correlation $\rho$ & Significance $^{\mathbf{a}}$ \\
\hline Metabolism & & \\
Energy metabolism & 0.23 & $* * *$ \\
Lipid metabolism & 0.49 & $*$ \\
Nucleotide metabolism & 0.75 & $*$ \\
Genetic information processing & & \\
Transcription & 0.16 & $* * *$ \\
Translation & 0.45 & $* * *$ \\
Folding, sorting and degradation & 0.46 & $* * *$ \\
Cellular processes & & $* * *$ \\
Transport and catabolism & 0.39 &
\end{tabular}

a Different biological pathways show considerable variability in their correlation of transcript-to-protein level changes. The statistical significance of the deviation from the global correlation $(\rho=0.61)$ was estimated by random sampling, controlling for the number of quantified proteins in each pathway $\left({ }^{*} P<0.05\right.$; $\left.{ }^{*} P<0.01 ;{ }^{* *} P<0.001\right)$.

For KEGG pathways involved in 'Metabolism', the correlations did not show a clear trend. The correlation was very high for 'Nucleotide metabolism' $(\rho=$ $0.75, N=105$, Figure $5 \mathrm{~A}$ ), indicating that changes of transcript expression cause corresponding changes in protein expression and, hence, only minor alterations in the post-transcriptional regulation occur between wild-type and tumor samples. In contrast, the correlation was low for 'Energy metabolism' ( $\rho=0.23, N=107$, Figure 5B). Here, however, transcript and protein expression levels were relatively constant and therefore no conclusions about changes in post-transcriptional regulation were possible.

The KEGG subcategories for 'Genetic information processing' generally showed significantly lower correlations. The correlation was low for 'Translation' ( $\rho=0.45, N=$ 298 , Figure $5 \mathrm{C}$ ) and lowest for genes regulating 'Transcription' ( $\rho=0.16, N=150$, Figure 5D). To control for the particular spread of mRNA and protein regulation, respectively, we compared the correlation of 'Transcription' genes to randomly sampled genes exhibiting similar spreads and found that the observed correlation was significantly lower than what would be expected by chance (data not shown). Thus, proteins involved in transcriptional processes are particularly well controlled on a post-transcriptional level, and changes of mRNA expression provide only limited insight into changes of protein expression. This is important to consider when performing quantitative research on the regulation of transcription: our data indicate that quantifying only mRNA expression might not always suffice to reflect the situation at the protein level. 
A

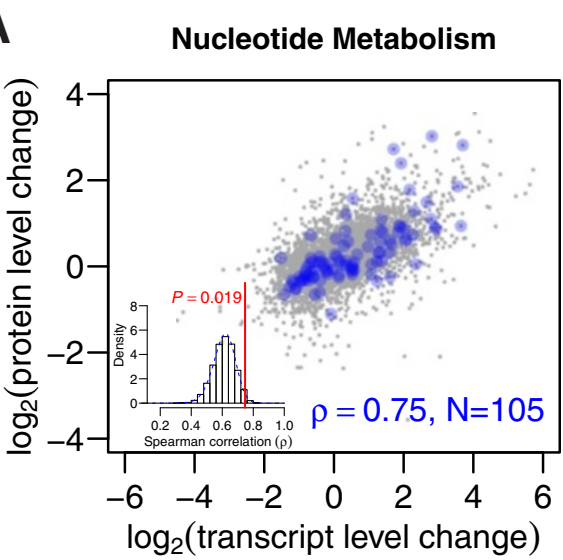

C

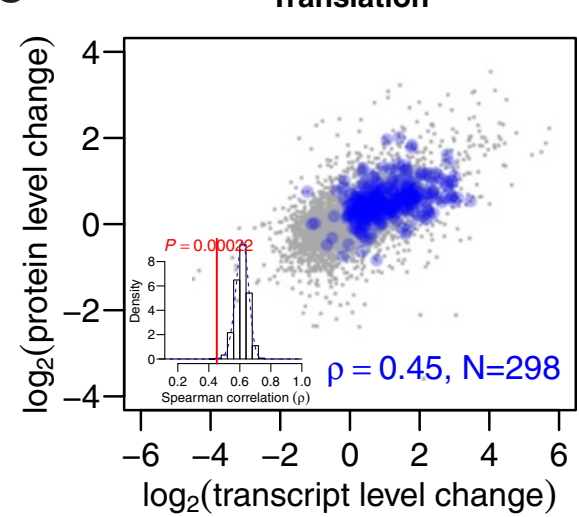

B

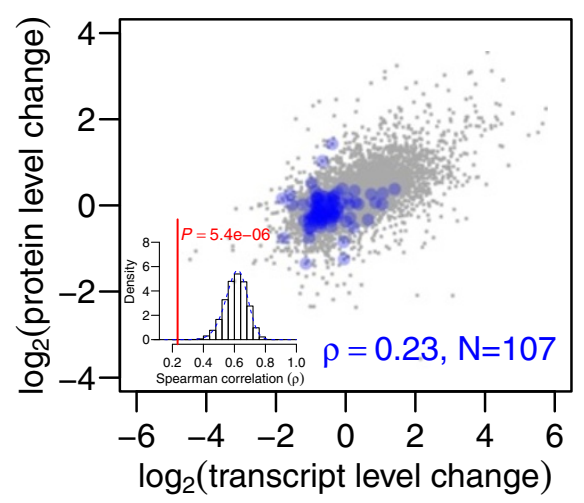

D

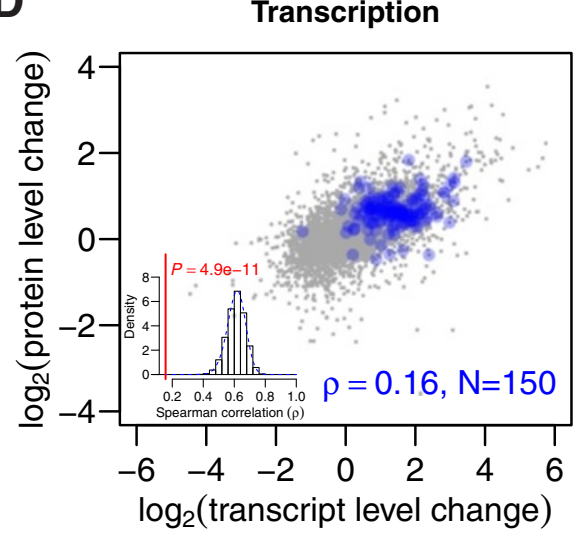

Figure 5 Post-transcriptional regulation changes for distinct functional pathways. Transcript and protein level changes exhibit different correlations for distinct gene functions. Correlation $\rho$ of transcript and protein level changes for genes involved in (A) nucleotide metabolism, (B) energy metabolism, (C) translation and (D) transcription. $N$ is the number of quantified gene products in each category. The insets on the lower left side of each panel show histograms of correlation coefficients that were generated by random sampling (sample size $\mathrm{N}$ ). The observed correlation coefficients and the corresponding $P$ values are indicated in red.

\section{Co-regulation of protein complexes is enhanced post-transcriptionally}

Most proteins exert their biological functions as part of supramolecular assemblies and complexes, and much progress has been made in identifying these protein complexes on a global scale [39-41]. While transcripts coding for protein-complex subunits tend to be co-expressed [42-44], co-regulation on the protein level has not been shown for large-scale data sets. This is important since a high degree of variation in subunit stoichiometries has been demonstrated for nuclear complexes, for example, see [45]. Also, little is known about the individual contributions of transcriptional and post-transcriptional mechanisms ensuring stoichiometric protein expression ratios.

We therefore used our quantitative transcriptome and proteome data to investigate the co-regulation of proteincomplex subunits. As a reference, we used all protein interactions and complexes identified by affinity purification of tagged proteins coupled with mass spectrometry and defined by the Drosophila protein interaction map (DPiM) [39]. Expression changes of transcripts and proteins were mapped onto the interaction network and visualized with Cytoscape [46]. Using this integrated protein interaction network (Figure 6 and Additional file 4), we observed a clustering of co-regulated genes into distinct areas corresponding to different protein complexes.

To confirm this co-regulation quantitatively, we determined the similarity of regulation between pairs of genes $A$ and $B$ by calculating the absolute difference of their $z$-transformed $\log _{2}$-fold expression changes $d_{A-B}$. Overall, we found that interacting proteins within a complex exhibited significantly higher co-regulation (that is, smaller $d_{A-B}$ ) than randomized protein pairs (Figure 7A). We observed a qualitatively similar effect for the transcripts (Additional file 5: Figure S5), but the co-regulation was significantly stronger at the protein than at the 


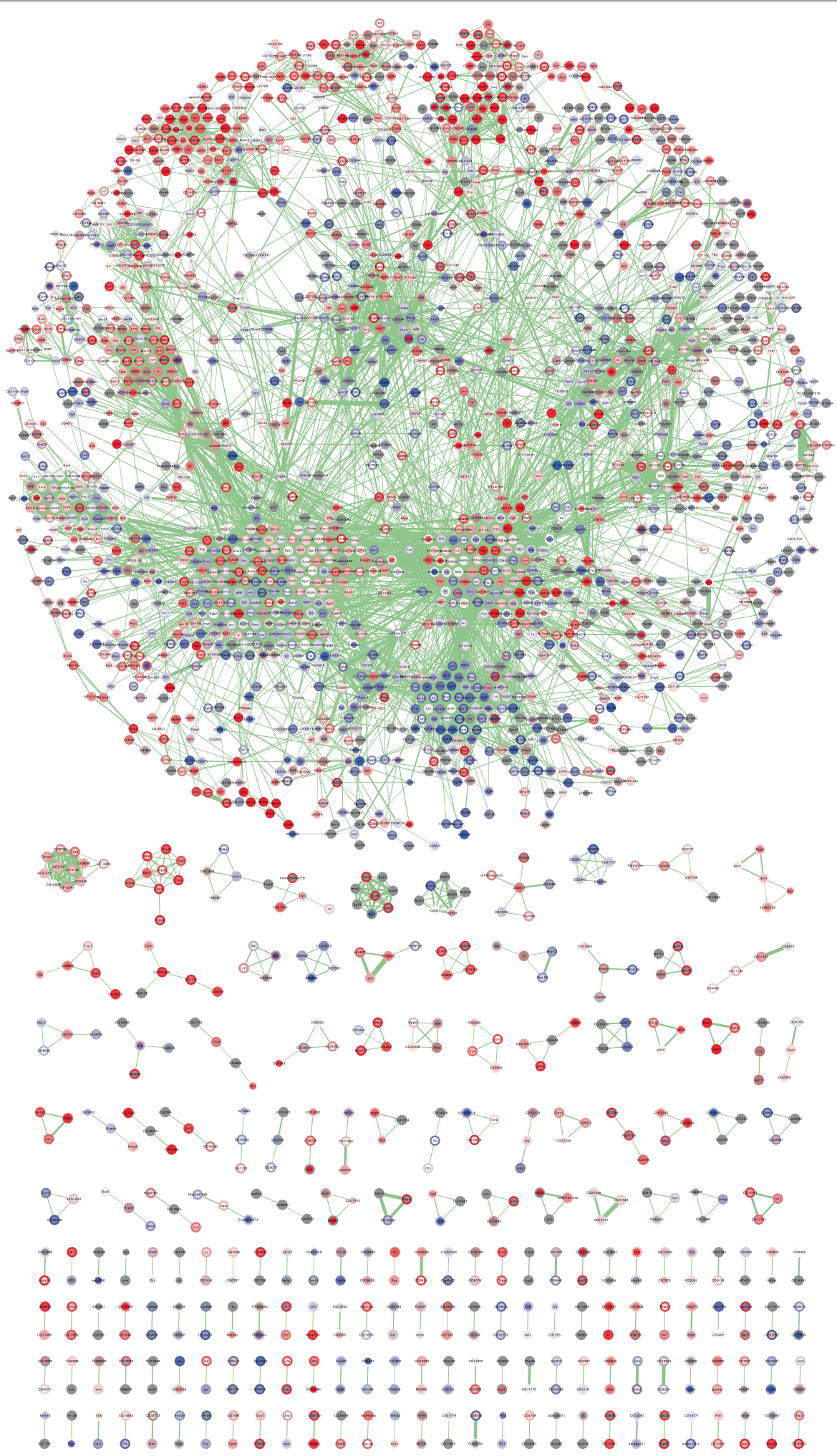

Figure 6 Global protein interaction map integrated with mRNA and protein expression changes. Visualization of mRNA and protein expression changes for all Drosophila protein interaction map clusters [39] using Cytoscape. Clustered proteins tend to be co-regulated. The centers of the nodes indicate protein expression changes and the borders of the nodes mRNA expression changes. Blue represents downregulation, red represents upregulation and the color intensity is proportional to the level of regulation. Transcripts and proteins not quantified are shown in gray. Protein interactions are depicted as light green lines and their thickness is proportional to the interaction strength. See Additional file 4 for details. 
mRNA level (Figure 7B). Taken together, our data indicate that despite co-regulation of complex subunits on the transcript level, significant fine-tuning of protein stoichiometry occurs post-transcriptionally.

\section{Only $11 \%$ of protein complexes exhibit significant subunit co-regulation}

In general, it is assumed that protein-complex subunits are co-regulated and maintain stable stoichiometric compositions. However, the distinction between permanent and transient complexes based on mRNA co-expression in yeast [44] and the discovery of variable subunit compositions for some nuclear complexes [45] indicate that there are exceptions to this rule.

To determine if the co-regulation we observed on the global level is due to co-regulation of all or of only a subset of protein complexes, we set out to identify the individual complexes that were co-regulated between wild-type and brat samples. For this, we compared the co-regulation of subunits of annotated protein complexes with randomly assembled 'complexes'. Surprisingly, we found that only 23 of 274 complexes were co-regulated on the transcript level. On the protein level, however, co-regulation was stronger and we identified 31 complexes exhibiting significant co-regulation (Figure 7C), supporting our conclusion for the post-transcriptional adjustment of protein stoichiometries and the higher importance of protein versus mRNA expression control.

The low fraction of co-regulated complexes $(11 \%$ on the protein level) indicates that either co-regulation is not a general feature of all protein complexes, or, more likely, that most complexes found in one biological system/state
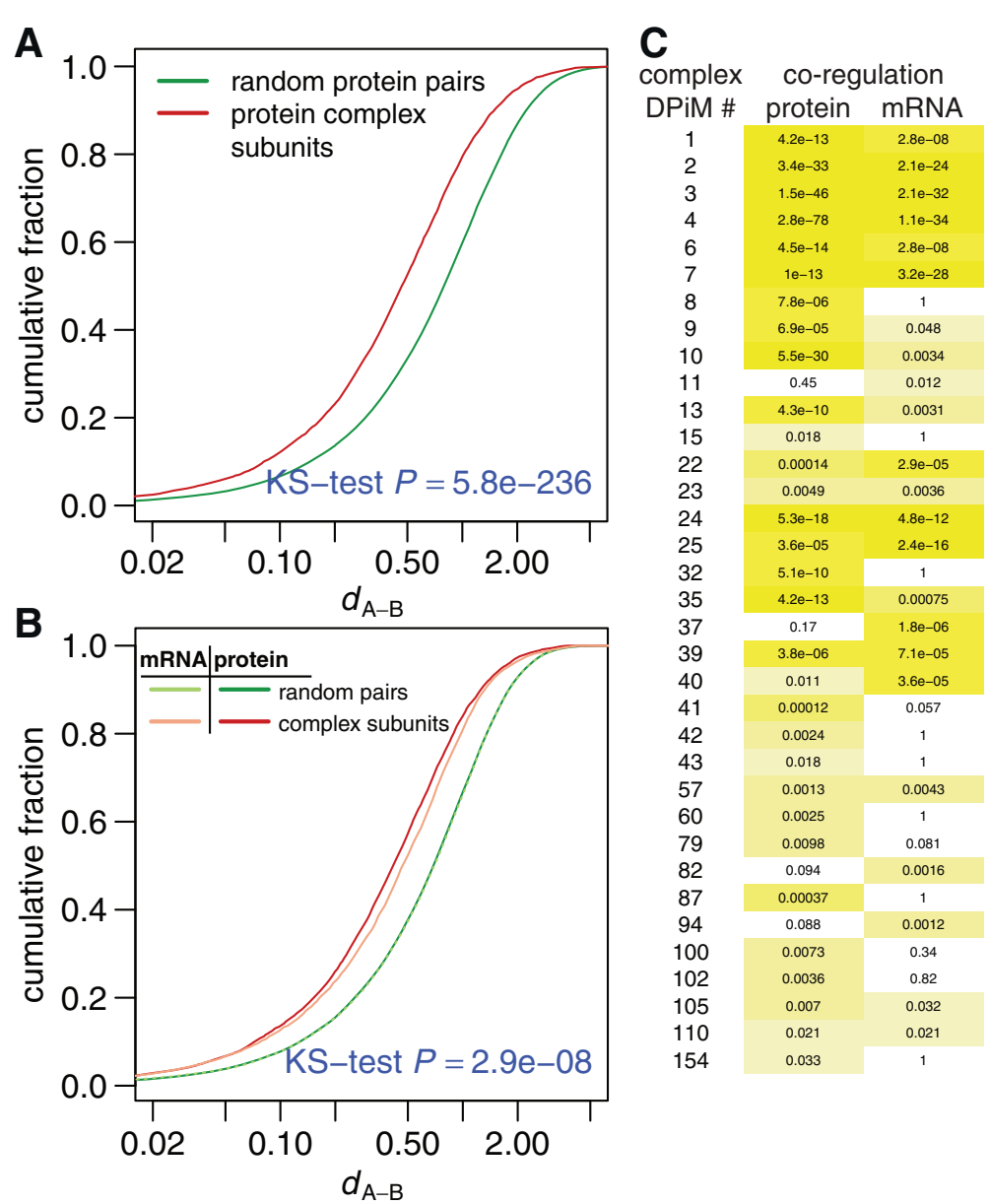

Figure 7 Post-transcriptional improvement of complex subunit co-regulation. (A) Subunits of annotated protein complexes (red) are significantly more co-regulated than random protein pairs (green). (B) Subunits of protein complexes are significantly more co-regulated on the protein level (red) than on the transcript level (light red). The random protein pairs are indicated in dark green, random transcript pairs in light green. (C) Matrix of $P$ values of significantly co-regulated protein complexes (DPiM) [39] at the mRNA level and at the protein level. The complexes are numbered according to DPiM since several of them have neither names nor known biological functions. The individual complex members are listed in Additional file 4. The color intensity indicates increasing significance. $P$ values were determined by the Kolmogorov-Smirnov test (KS-test) and corrected for multiple testing. DPiM, Drosophila protein interaction map. 
do not necessarily exist in other systems/states or exhibit different subunit compositions [45]. In addition, different molecules of a protein could be subunits of different protein complexes at a time.

As all annotated complexes were isolated from cultured cells of late embryonic origin (S2R+ cells) [39], and, since they exhibited high co-regulation in adult brain tissue, we propose to classify them as permanent core complexes of Drosophila. Complexes like the proteasome (DPiM \#4), the SNAP/SNARE complex (DPiM \#7), the eukaryotic initiation factor 3 complex (eIF3, DPiM \#24) and the ATP synthase complex (DPiM \#25) fall into this category, both at the mRNA level and at the protein level, whereas the exosome (DPiM \#41), the prefoldin complex (DPiM \#42), the TCP-1 ring complex or chaperonin-containing TCP1 complex (TRiC/CCT, DPiM \#32) and the minichromosome maintenance complex (MCM, DPiM \#60) are exclusively co-regulated on the protein level.

Given this high variability, we would like to suggest an extension of the concept of permanent and transient complexes introduced by [44] because higher organisms are characterized by having different tissue types and specific developmental programs. In this situation, many more complexes have to be characterized as dynamic or transient, since they might only occur at specific times, places or physiological states during an organism's lifetime.

\section{Characterization of individual complexes in a tumor based on subunit co-regulation}

In total, our analysis defined 31 co-regulated core complexes (Figure 7C). From those, we selected a subset of well-known complexes and manually re-analyzed their annotated subunit composition as well as their potential for tumorigenesis taking into account expression changes at the mRNA level and at the protein level.

The eIF3 complex (DPiM \#24, Figure 8A) is essential for the assembly of the translation initiation machinery, namely the recruitment of initiator Met-tRNAi and mRNA to the $40 \mathrm{~S}$ ribosome, and the subsequent scanning for the AUG start codon [47]. Aberrant mRNA and protein levels of eIF3 subunits have been detected in a wide variety of solid tumors and cancer cell lines, and eIF3 overexpression can promote malignant transformation (see [48] and references therein). We found eIF3 mildly but consistently upregulated in brain tumors at the transcript as well as at the protein level.

The ATP synthase complex (DPiM \#25, Figure 8B) is involved in the oxidative phosphorylation pathway and employs the electrochemical gradient at the inner mitochondrial membrane for generating ATP from ADP. The downregulation of oxidative phosphorylation is a wellknown metabolic hallmark of cancer cells, called the Warburg effect $[49,50]$. In the brat tumors, ATP synthase was downregulated both at the transcript level and protein level. Interestingly, the glycolytic enzyme L-lactate dehydrogenase (ecdysone-inducible gene L3, ImpL3 in Drosophila) was about twofold upregulated suggesting that Drosophila tumors might provide a suitable model for analyzing the causal relationships of the Warburg effect with cancer progression. Knockdown of this enzyme has been shown to increase mitochondrial respiration and to attenuate tumor growth [51,52].

The MCM complex (DPiM \#60, Figure 8C) functions as a replicative helicase. It unwinds duplex DNA and enables fork progression during DNA replication [53]. We found all six complex members strongly upregulated both at the transcript level and at the protein level. Coaffinity purification identified an additional member of the MCM complex, the previously uncharacterized protein CG3430 [39]. Our data show the co-regulation of CG3430 with the other six MCM complex members, hence supporting this assignment.

The exosome complex (DPiM \#41, Figure 8D) is required for 3' $\rightarrow$ ' RNA processing and turnover [54]. All its subunits were upregulated; however, the upregulation was generally stronger on the mRNA level than on the protein level. Together with the observation that the exosome subunits are significantly co-regulated at the protein but not the transcript level (Figure 7C), this suggests, that post-transcriptional mechanisms might be involved in regulating exosome expression. It has been shown that the exosome interacts and co-localizes with the essential elongation factor Spt6 at active chromatin, indicating that the exosome might exert its pre-mRNA surveillance function co-transcriptionally [55]. Our data support this interaction by demonstrating the co-regulation of Spt6 with the exosome.

The TRiC/CCT complex (DPiM \#32, Figure 8E) is an essential, ATP-dependent chaperonin consisting of two identical stacked rings with eight paralogous subunits per ring. It interacts with about $10 \%$ of newly synthesized cytosolic proteins and prevents the accumulation of toxic aggregates [56,57]. Guruharsha et al. identified six of the eight known TRiC/CCT complex members plus three weakly connected proteins (CG3313, shd, viaf), whereas the remaining two core subunits, CCT $\gamma$ and Tcp- $1 \zeta$, were assigned to the DPiM clusters $\# 8$ and \#28, respectively [39]. According to our mRNA and protein data, however, the co-regulation of $\mathrm{CCT} \gamma$ and Tcp- $1 \zeta$ was most consistent with them belonging to the TRiC/CCT complex (Figure 8F). For the three weakly associated proteins, we were not able to detect CG3313 nor shd, but viaf was co-regulated with the other complex members. Consistent with the upregulation of the TRiC/CCT complex in brain tumors, we have previously shown that tissue-specific knockdown of subunits by RNAi leads to under-proliferation or death of neuroblasts [58]. 




The TRiC/CCT complex is recruited to nascent chains by the multisubunit chaperone complex prefoldin (DPiM \#42, Figure 8G) allowing co-translational folding of proteins [57]. Three new subunits have been proposed for the prefoldin complex: CG8617, CG9542 and CG10252 [39]. We were not able to identify CG8617 nor CG10252 on the protein level, and CG9542 exhibited a strong downregulation unlike the behavior of the established complex members. Therefore, we propose that these prefoldin interaction partners are cell-type-specific subunits of the complex and are most likely not an integral part of the prefoldin complex in adult female fly heads.

Taken together, we have shown that co-regulation data at the mRNA level and at the protein level provide 
valuable additional information for protein-complex assignment, especially if protein-complex data is to be used for different experimental systems.

\section{Conclusions}

We have compiled a comprehensive data set of tissuespecific expression changes that occur in a tumor model both on the transcriptome and on the proteome level (Additional file 6). In our integrative analysis we use this data set and demonstrate the impact of posttranscriptional gene regulation for different biological processes and protein complexes.

To achieve iTRAQ quantification for $70 \%$ of all expressed protein-coding genes in a complex tissue, we employed: (1) digestion with two proteases to produce largely non-overlapping peptides, (2) high-resolution chromatography and fractionation to reduce sample complexity and (3) different mass spectrometry fragmentation techniques to obtain optimal quantitative information. This protocol is in principle applicable to clinical samples, since it does not require in vivo labeling. Our data set covers $86 \%$ of the expressed proteins in the Drosophila head annotated to distinct biological pathways (Figure 3C).

At the global level, we provide evidence for a general regulatory function of the transcript UTRs, that is, shorter 3' and 5' UTRs lead to increased protein upregulation. This finding indicates that regulatory elements in the UTRs ensure proper protein expression control and that transcripts with longer UTRs are less prone to misexpression, potentially due to the dampening presence of binding sites for RNA-interacting proteins or miRNAs.

Overall, alterations in transcript and protein expression are well correlated. However, distinct biological processes show highly different correlations. This suggests that posttranscriptional regulation strongly affects some processes like transcription whereas other processes like nucleotide metabolism are barely affected (Figure 5). The differential effects of post-transcriptional regulation in wild-type and tumor tissue should be taken into account when analyzing transcriptome data and, in addition, might offer new directions for targeted tumor treatment.

By integrating our data set with protein-complex information [39], we have compiled one of the first systemslevel networks for the dynamics of protein complexes (Figure 6). By statistically investigating the co-regulation of protein-complex subunits we show that complex stoichiometry is ensured by both transcriptional and posttranscriptional contributions, and that co-regulation on the protein level is more stringently controlled than on the mRNA level. The function of many complexes critically depends on the proper stoichiometric presence of all subunits, and the consequences of the misexpression of any one subunit can range from wasting energy to dominant negative effects and diseases. Therefore, this two-tiered mechanism is important for controlling the relative abundance of protein-complex subunits. Surprisingly, however, we find that only a small fraction of protein-complex subunits is co-regulated in the Drosophila brain suggesting that complexes exhibit high degrees of context-dependent dynamics.

The analysis of our integrative network shows that several protein complexes are consistently deregulated in tumors, and we find preliminary evidence for alterations reminiscent of the Warburg effect (Figure 8B). We provide examples for which the co-regulation of potential proteincomplex subunits contributes valuable additional information for assigning subunits to the correct complexes. Since large-scale protein interaction analyses are usually performed in cell culture systems, our approach could be used to re-evaluate this information in a tissue-specific context.

In summary, besides providing a valuable resource for further system-wide studies and quantitative biology, our data offers novel insights into characteristic alterations of tumor gene expression and post-transcriptional gene regulation.

\section{Materials and methods Sample preparation}

All Drosophila stocks were maintained and crossed at $22^{\circ} \mathrm{C}$ according to standard procedures. The brat allele brat ${ }^{k 06028}$ was acquired from the Bloomington Drosophila Stock Center (Indiana) and balanced over $\mathrm{CyO}, P\{h s$-hid $\}$ to allow us to select homozygous mutant offspring using a heat shock. For transcriptome and proteome analysis, adult female flies that were 1 to 3 days old were collected manually (wild-type control and $b r a t^{k 06028}$ ). Flies were transferred into $15 \mathrm{ml}$ conical tubes and snap-frozen in liquid nitrogen. Heads were separated by vigorously shaking and vortexing the tubes for $30 \mathrm{~s}$ and then applying the mixture to a stack of sieves $(800,590,355 \mu \mathrm{m}$ mesh openings) submerged in liquid nitrogen. The isolated heads were transferred into microfuge tubes and stored at $-80^{\circ} \mathrm{C}$ for further analysis. Fly heads were ground to a powder using a mortar and pestle cooled with liquid nitrogen.

\section{Transcriptome sequencing}

Total RNA was isolated from adult fly heads by TRIzol purification (Invitrogen), and genomic DNA was removed using gDNA eliminator columns from the RNeasy Mini Kit (Qiagen) following the manufacturer's instructions. RNA quality was assessed by spectrophotometry (NanoDrop, Thermo Fisher Scientific) and on a Bioanalyzer (Agilent). The RNA was enriched for poly(A)+ mRNA (Dynabeads mRNA purification kit, Invitrogen), fragmented and subjected to first-strand cDNA synthesis (based on a protocol by [59]). After second-strand 
cDNA synthesis, double-stranded cDNA was purified and quantified. The library was prepared using a modified protocol from Illumina with NEBNext DNA sample Prep Reagent kits (NEB). Double-stranded cDNA was end-repaired, poly(A) was added and adapters were ligated to DNA fragments. After size selection (200 to $600 \mathrm{bp}$ ), and UDGase-treatment for strand specificity, adapter-modified DNA fragments were enriched by PCR. Next, 76-base paired-end sequencing was performed on a Genome Analyzer IIx (Illumina).

The strand-specific paired-end reads were screened for ribosomal RNA by alignment (maximum of three mismatches) against known rRNA sequences (RefSeq) using Bowtie [60]. The insert statistics were estimated by aligning the remaining reads uniquely to the transcriptome and calculating the mean insert length and standard deviation. The rRNA-subtracted pairedend reads were aligned with TopHat [61] against the Drosophila melanogaster genome (release 5). Introns of 30 to 150,000 bp were allowed based on FlyBase statistics. Maximum multihits was set to 1 , and microexonsearch was enabled. Additionally, a gene model was provided as gene transfer format (GTF) file (Ensembl BDGP5.25.60). Aligned reads in valid pairs were subjected to FPKM estimation using Cufflinks $[62,63]$. Bias detection and correction were performed in this step. Furthermore, only those fragments compatible with Ensembl annotation (BDGP5.25.60) were allowed and counted towards the number of mapped hits used in the FPKM denominator.

The transcriptome data have been deposited in the NCBI Gene Expression Omnibus and are accessible through GEO Series accession number GSE51412.

\section{Protein digestion and peptide iTRAQ labeling}

Fly-head powder was resuspended in $8 \mathrm{M}$ urea/0.1 M triethylammonium bicarbonate (TEAB) and centrifuged to remove insoluble material. Proteins in the supernatant fraction were precipitated by the addition of ice-cold acetone and incubated at $-80^{\circ} \mathrm{C}$ overnight. After centrifugation, pellets were resuspended in iTRAQ dissolution buffer (0.5 M TEAB) containing 1\% RapiGest (Waters), and the protein concentration was determined using the BCA Protein Assay Kit (Pierce). Cysteine residues were reduced and alkylated using tris-(2-carboxyethyl)phosphine (TCEP) and methyl methanethiosulfonate (MMTS) according to the instructions for the iTRAQ labeling kit (Applied Biosystems). Samples were digested with trypsin or LysC at $37^{\circ} \mathrm{C}$ for $16 \mathrm{~h}$ and labeled separately with iTRAQ 4-plex reagents according to the manufacturer's instruction, using 100 $\mu \mathrm{g}$ peptides for each label. A duplicate labeling strategy was pursued, that is, each sample was labeled with two different tags.

\section{Two-dimensional fractionation of labeled peptides}

Labeled peptides from four samples were combined, acidified with formic acid (FA), lyophilized, resuspended in $5 \mathrm{mM}$ sodium phosphate buffer ( $\mathrm{pH}$ 2.7) containing 15\% acetonitrile (ACN), and injected onto a Polysulfoethyl-A $3 \mu \mathrm{m}$ (PolyLC), $25 \mathrm{~cm} \times 1 \mathrm{~mm}$ inner diameter (i.d.) column. Separation was performed on an UltiMate nano LC system (Dionex, Thermo Fisher Scientific) at a flow rate of $50 \mu \mathrm{l} / \mathrm{min}$ using the following gradient: $20 \mathrm{~min} 100 \%$ $\mathrm{A}$, followed by a linear gradient to $10 \% \mathrm{~B} / 50 \% \mathrm{C}$ in 80 min, $25 \% \mathrm{~B} / 50 \% \mathrm{C}$ in $10 \mathrm{~min}, 50 \% \mathrm{~B} / 50 \% \mathrm{C}$ in $5 \mathrm{~min}$ and maintained for a further $15 \mathrm{~min}$, then within $5 \mathrm{~min}$ to $100 \% \mathrm{~A}$ for column re-equilibration (A: $5 \mathrm{mM}$ sodium phosphate buffer, $15 \% \mathrm{ACN}, \mathrm{pH}$ 2.7; B: $5 \mathrm{mM}$ sodium phosphate buffer, $0.5 \mathrm{M} \mathrm{NaCl}, 15 \% \mathrm{ACN}, \mathrm{pH} 2.7$; C: $5 \mathrm{mM}$ sodium phosphate buffer, $15 \% \mathrm{ACN}, \mathrm{pH} 6.0$ ). One-minute fractions were collected and fractions with a low peptide content were pooled. Samples were concentrated in a vacuum centrifuge concentrator to remove $\mathrm{ACN}$ and diluted in $0.1 \%$ trifluoroacetic acid (TFA). Reversed phase separation was performed on an UltiMate 3000 RSLCnano high performance liquid chromatography (HPLC) system (Dionex, Thermo Fisher Scientific). After injection, samples were concentrated and desalted on a trapping column (AcclaimPepMap $3 \mu \mathrm{m}, 100 \AA, 2 \mathrm{~cm} \times 75 \mu \mathrm{m}$ i.d.) using $0.1 \%$ TFA at a flow rate of $5 \mu \mathrm{l} / \mathrm{min}$ as a loading solution, and then separated on an analytical column (AcclaimPepMap $2 \mu \mathrm{m}, 100 \AA ⿻ 25 \mathrm{~cm} \times 75 \mu \mathrm{m}$ i.d.) using a linear gradient from $2 \%$ to $25 \%$ B in $175 \mathrm{~min}$, then to $90 \%$ B in $5 \mathrm{~min}$, maintained for $5 \mathrm{~min}$, then within $2 \mathrm{~min}$ to $100 \%$ A for column re-equilibration (A: $2 \% \mathrm{ACN} / 0.1 \% \mathrm{FA}$; B: $80 \% \mathrm{ACN} / 10 \%$ trifluoroethanol $/ 0.08 \% \mathrm{FA})$ at a flow rate of $275 \mathrm{nl} / \mathrm{min}$. The HPLC was directly coupled online to a LTQ-Orbitrap Velos instrument (Thermo Fisher Scientific) via a nanoelectrospray source (Proxeon, Thermo Fisher Scientific).

\section{Shotgun mass spectrometry}

The LTQ-Orbitrap Velos instrument was operated in positive ionization mode. The source voltage was set to $2.0 \mathrm{kV}$, transfer tube temperature was $250^{\circ} \mathrm{C}$ and the S-lens radio frequency (RF) level was set to $68 \%$. A mass spectrometry (MS) survey scan was performed in the Orbitrap from a mass-to-charge ratio $(\mathrm{m} / z)$ of 350 to 2,000 at a resolution of 60,000 . The automatic gain control (AGC) target value was set to 1,000,000 ions and the maximum fill time was 500 ms. The lock mass option was enabled using the dimethylcyclosiloxane background ions (protonated $\left.\left[\left(\mathrm{CH}_{3}\right)_{2} \mathrm{SiO}\right]_{6} ; m / z=445.120025\right)$ for internal calibration. The MS survey scan was followed by 12 data-dependent scans.

The six most abundant ions excluding singly charged ions were selected for fragmentation. For each selected precursor ion, two tandem mass spectra were obtained: 
one spectrum was acquired in the ion trap for maximum sensitivity of identification and the other spectrum was acquired in the Orbitrap at a resolution of 7,500 (AGC target 100,000, maximum fill time $250 \mathrm{~ms}$ ) for precise quantification. The strategy is analogous to the previously described method, which combined a CID spectrum acquired from the ion trap with a HCD spectrum acquired from the Orbitrap [28]. However, for samples digested with trypsin a CID spectrum was recorded in the ion trap, while for samples digested with LysC an ETD spectrum was recorded using the ion trap. For CID spectra, the AGC target was set to 6,000 ions, maximum fill time was $200 \mathrm{~ms}$, activation time was $10 \mathrm{~ms}$, normalized collision energy was $35 \%$, and multistage activation was activated using the following neutral loss $\mathrm{m} / z$ list: 32.6, 49.0 and 98.0. For ETD spectra, the AGC target was set to 10,000 ions, maximum fill time was $200 \mathrm{~ms}$, supplemental activation was enabled, and the reaction time was set to 120 $\mathrm{ms}$ for doubly charged precursor ions and $80 \mathrm{~ms}$ for triply charged precursor ions, reduced in a charge-dependent manner for higher charged precursor ions. Reagent ion target was 300,000 ions with a maximum fill time of 80 $\mathrm{ms}$ for ETD, and the reagent ion source chemical ionization $(\mathrm{CI})$ gas pressure was tuned whenever indicated to ensure adequate fluoranthene signal. For HCD spectra, a stepped collision energy was employed with two steps at $42 \%$ and $58 \%$ normalized collision energy to permit both identification and quantification.

To fragment peptides close to the apex of the elution signal, the chromatography feature was activated using a correlation of 0.8 and an expected peak width of 10 s. In all cases, one microscan was recorded. The isolation window was $2.4 \mathrm{~m} / z$ for spectra recorded in the ion trap and $1.6 \mathrm{~m} / \mathrm{z}$ for spectra recorded in the Orbitrap to minimize interference with iTRAQ quantification by precursor ions with similar $m / z$. To avoid oversampling, the $m / z$ values of precursor ions selected for fragmentation were subsequently excluded for $180 \mathrm{~s}$ using a dynamic exclusion window of $\pm 5 \mathrm{ppm}$, with the early expiration feature deactivated. Database searches were performed on both ion trap and Orbitrap tandem mass spectra for identification while quantification was based on iTRAQ reporter ions extracted from Orbitrap tandem mass spectra. Measurements were started with SCX fractions separated from one another by $10 \mathrm{~min}$ of elution time during strong cation exchange separation. Subsequently, adjacent SCX fractions were measured using exclusion lists of peptides identified in adjacent SCX fractions to maximize proteome coverage. The exclusion lists were based on a retention time window of $2 \mathrm{~min}$ before and $4 \mathrm{~min}$ after the retention time of the peptide identified in the adjacent SCX fraction, the exact theoretical $m / z$ of the identified peptide and a tolerance window $\pm 7.5 \mathrm{ppm}$. To make efficient use of the exclusion lists, monoisotopic precursor selection was enabled and preview mode was deactivated.

The mass spectrometry proteomics data have been deposited with the ProteomeXchange Consortium [64] via the PRIDE partner repository [65] with the data set identifier PXD000478.

\section{Data analysis}

Data generated by LC-MS/MS analysis were searched against a database containing a translation of all open reading frames in FlyBase (r5.25) [66] and common contaminants, concatenated to a reversed decoy database so that the FDR could be estimated using the target-decoy strategy [67]. Proteome Discoverer (version 1.3.0.211, Thermo Fisher Scientific) was used as a search engine interface for Mascot [68], Sequest [69], X!-Tandem [70] and ZCore [71]. Oxidation of methionine was set as dynamic modification, and methylthio $(C)$ and iTRAQ4plex label (K, N-terminus) as static modifications. The minimal peptide length was set to seven amino acids, and a maximum of two missed cleavages was allowed for trypsin- and LysC-digested samples. To allow for an integrative analysis of transcriptome and proteome data, protein level changes were determined using only peptides that mapped unambiguously to one gene. Peptides that could be derived from proteins encoded by different gene models ('shared peptides') were excluded [72]. Furthermore, only peptides that showed less than a twofold difference between duplicate iTRAQ channels were included in the analysis. Peptide identifications from different search engines were combined using a modified version of the combined FDR score [73]. Reporter ion intensities were corrected for isotope impurities in the iTRAQ labels. To account for the error structure and stabilize the variance of the reporter ion intensities, a variance stabilizing transformation was applied [74]. Protein ratios were calculated as the $20 \%$ trimmed mean from the median-centered peptide ratios [74]. Proteins were filtered for a maximum FDR of $5 \%$ [75].

\section{Selected reaction monitoring assays}

To validate iTRAQ quantification using an independent label-free method, SRM assays were performed on selected proteins. Suitable peptides were selected from either the iTRAQ data set or the Peptide Atlas [76] or were predicted in silico. The selection process was aided by MRM Pilot Software (AB Sciex). In addition, three peptides each from four different proteins (fructosebisphosphate aldolase [UniProt:P07764], heat shock protein $70 \mathrm{kDa}$ [UniProt:P11147], enolase [UniProt:P15007] and phosphoglycerate kinase [UniProt:Q3KN29]) that were found unregulated in the iTRAQ data set were used to normalize the runs from wild-type and brat samples. Unlabeled protein extracts were separated by 
one-dimensional reversed-phase nanoHLPC on an Ultimate 3000 (Thermo Fisher Scientific). Samples were loaded onto a trapping column (PepMap C18, $5 \mu \mathrm{m}, 100$ $\AA, 5 \mathrm{~mm} \times 0.3 \mathrm{~mm}$ i.d.) using $0.1 \%$ TFA at a flow rate of $20 \mu \mathrm{l} / \mathrm{min}$ and desalted for $20 \mathrm{~min}$. Peptides were separated on a $250 \mathrm{~mm} \times 75 \mu \mathrm{m}$ i.d. analytical column (PepMap C18, $3 \mu \mathrm{m}, 100 \AA$ ) at a flow rate of $300 \mathrm{nl} / \mathrm{min}$ by applying the following gradient: in $130 \mathrm{~min}$ from 0 to $100 \% \mathrm{~B}$, in $30 \mathrm{~min}$ to $100 \% \mathrm{C}$, held for $5 \mathrm{~min}$ at $100 \% \mathrm{C}$ before re-equilibration with $100 \% \mathrm{~A}$ (A: $5 \% \mathrm{ACN}$, $0.1 \%$ FA; B: $30 \%$ ACN, $0.1 \%$ FA; C: $80 \%$ ACN, $10 \% 2,2$, 2-trifluoroethanol, $0.08 \%$ FA). The nanoLC was directly coupled to a QTRAP4000 hybrid triple quadrupole/linear ion-trap instrument (Applied Biosystems). Transitions were validated via MS2 spectra and the best two to three transitions per peptide were selected for quantification. Peptides that could not be verified by MS2 were synthesized in-house on a Syro Peptide Synthesizer (MultiSyntech) and used as standards to determine retention time and optimal transitions. For quantification runs, $2 \mu \mathrm{g}$ of unlabeled protein extract was injected and the mass spectrometer was operated in SRM mode without acquisition of MS2 spectra. Transitions were monitored either with a fixed dwell time of $100 \mathrm{~ms}$ for candidate proteins and $50 \mathrm{~ms}$ for normalization peptides, or by scheduled SRM. Peak area integration was done with the MultiQuan 1.0 software (Applied Biosystems).

\section{Bioinformatics}

All statistical analyses were performed using the $\mathrm{R}$ programming language [77]. Primary sequence features like codon adaptation index, molecular weight and isoelectric point were calculated using EMBOSS applications [78]. Hydrophobicity was calculated according to [79]. For every gene, the longest FlyBase-annotated 5' UTR, coding sequence and 3' UTR were used. The hypergeometric test was applied for KEGG enrichment analysis and corrected for multiple testing.

To determine if the correlations between transcript and protein level changes for different biological pathways were significantly different from the global correlation, random sampling experiments were performed with the sample size matching the number of proteins quantified in each pathway. For every pathway, a skew-normal distribution was fitted to the Spearman correlation coefficient distribution of 10,000 random samples and the $P$ value was estimated [80].

To control for the different spread of regulation in different pathways on the transcript or protein level, the gene list was rank-ordered according to transcript or protein level change, respectively, and random samples were drawn out of the 20 closest neighbors of each gene in the pathway under investigation, thereby maintaining the pathway-specific range of regulation. The correlation coefficients of 10,000 random samples were calculated and a skew-normal distribution was fitted to estimate the $P$ value.

Transcript and protein level changes were expressed as $\log _{2}$-fold changes and $z$-transformed. For co-regulation analysis, transcript and protein $z$ values were quantile normalized. Co-regulation between two genes $A$ and $B$ was expressed as the absolute difference between their normalized $z$ values $d_{A-B}$, with small values indicating co-regulation. This value measured the co-regulation of complex subunits irrespective of their stoichiometric composition. We define a protein complex as consisting of at least three different subunits, and we distinguish two categories of protein-protein interactions: random protein pairs that generally do not interact and interacting proteins that are members of the same protein complex.

\section{List of abbreviations}

ACN, acetonitrile; AGC, automatic gain control; bp, base pair; CDS, coding sequence; CID, collisional-induced dissociation; DPiM, Drosophila protein interaction map; eIF3, eukaryotic initiation factor 3; ETD, electron transfer dissociation; FA, formic acid; FDR, false discovery rate; FPKM, fragments per kilobase of transcript per million mapped fragments; $\mathrm{HCD}$, higher energy C-trap dissociation; iTRAQ, isobaric tag for relative and absolute quantification; KEGG, Kyoto Encyclopedia of Genes and Genomes; LC-MS/MS, liquid chromatography-tandem mass spectrometry; MCM, minichromosome maintenance complex; miRNA, microRNA; PCR, polymerase chain reaction; rRNA, ribosomal RNA; SCX, strong cation exchange; SRM, selected reaction monitoring; TEAB, triethylammonium bicarbonate; TRiC/CCT, TCP-1 ring complex or chaperonin-containing TCP-1 complex; UTR, untranslated region.

\section{Additional files}

Additional file 1: Figure S1. iTRAQ reproducibility. (A) Technical reproducibility of iTRAQ protein quantification. (B) Technical reproducibility between ITRAQ and label-free SRM protein quantification. Error bars indicate standard deviations. (C) Reproducibility of protein level changes between biological replicates measured with SRM and iTRAQ. Error bars indicate standard deviations. (D) Venn diagram showing the number of quantified unique peptides in the trypsin-and LysC-digested samples. The samples were largely complementary: only $16 \%$ of the quantified peptides were identical. (E) Venn diagram showing the number of quantified proteins from the trypsin and LysC samples. This shows that $75 \%$ of the proteins were quantified in both samples. (F) Correlation of iTRAQ protein quantification using either trypsin- or LysC-digested samples.

Additional file 2: Figure S2. Analysis of proteome coverage. For each bin the number of annotated (dark gray), expressed (light gray) and quantified proteins (white) are shown together with the percentage of quantified proteins (red). (A) Proteome coverage is higher for proteins predicted by the codon adaptation index to be more abundant. The blue line indicates 
the percentage of quantified proteins from all annotated protein. (B) Proteome coverage is higher for larger proteins. (C) Proteome coverage is lower for very hydrophobic proteins. (D) Proteome coverage is higher than $60 \%$ for all isoelectric points.

Additional file 3: Figure S3. Correlation of protein level change with transcript abundance. Correlation of protein level change with transcript abundance in (A) wild-type and (B) brat samples.

Additional file 4: Cytoscape protein interaction network. Cytoscape file containing $\log _{2}$-fold expression changes on mRNA and protein levels combined with DPiM protein interaction data. The centers of the nodes indicate protein expression changes and the borders of the nodes mRNA expression changes. Blue represents downregulation, red represents upregulation and the color intensity is proportional to the level of regulation. Transcripts and proteins not quantified are shown in gray. Protein interactions are depicted as light green lines and their thickness is proportional to the interaction strength.

Additional file 5: Figure S5. Complex co-regulation. Protein-complex co-regulation on the mRNA level. Transcripts encoding subunits of annotated protein complexes (red) are significantly more co-regulated than random pairs (green).

Additional file 6: Data set of transcriptome and proteome changes. Complete data set of transcript and protein quantification data, containing FlyBase gene number, gene name, protein level change ( $\log _{2}$-fold change), standard deviation of $\log _{2}$ protein level change, number of quantified spectra, transcript level change ( $\log _{2}$-fold change), brat FPKM, control FPKM, standard deviation of brat FPKM and standard deviation of control FPKM. Since a double-labeling approach was performed, each quantified spectrum contains two reporter ions from both brat and control samples.

\section{Competing interests}

The authors declare that they have no competing interests.

\section{Authors' contributions}

$\mathrm{CJ}$ and JAK conceived the project. CJ performed most of the experiments and analyzed the data. ID and PP generated the proteomics data. HH generated the transcriptome data. GA and KM designed and supervised the mass spectrometry experiments. RS developed the chromatography procedure. $\mathrm{CJ}$ and JAK wrote the manuscript. All authors read and approved the final manuscript.

\section{Acknowledgements}

We are grateful to members of the Knoblich and Mechtler labs for helpful discussions. We thank T. Burkard, C. Esk and T. Köcher for comments on the manuscript. We thank T. Burkard for help with the RNA-seq data, and M. Mazanek and G. Krssáková for technical support with LC-MS/MS. Deep sequencing was performed at the CSF NGS Unit (csf.ac.at). CI was supported by a FEBS long-term fellowship. Work in KM's laboratory is supported by the Austrian Science Fund FWF (Fonds zur Förderung der wissenschaftlichen Forschung) SFB-F3402 Chromosome Dynamics, and the European Commission via the FP7 projects MEIOsys and Prime-XS. Work in JAK's laboratory is supported by the Austrian Academy of Sciences, the Austrian Science Fund FWF (projects P20547-B09, Z153-B09 and 1552-B19) and an advanced grant from the European Research Council.

\section{Author details}

${ }^{1}$ Institute of Molecular Biotechnology of the Austrian Academy of Sciences (IMBA), Dr Bohr-Gasse 3, 1030 Vienna, Austria. ${ }^{2}$ Christian Doppler Laboratory for Proteome Analysis, Dr Bohr-Gasse 9, 1030 Vienna, Austria. ${ }^{3}$ Research Institute of Molecular Pathology (IMP), Dr Bohr-Gasse 7, 1030 Vienna, Austria. ${ }^{4}$ Thermo Fisher Scientific, Abberdaan 114, 1046 AA Amsterdam, Netherlands.

Received: 12 July 2013 Accepted: 30 November 2013

Published: 30 November 2013

\section{References}

1. Komili S, Silver PA: Coupling and coordination in gene expression processes: a systems biology view. Nat Rev Genet 2008, 9:38-48.
2. de Sousa Abreu R, Penalva LO, Marcotte EM, Vogel C: Global signatures of protein and mRNA expression levels. Mol Biosyst 2009, 5:1512-1526.

3. Maier T, Güell M, Serrano L: Correlation of mRNA and protein in complex biological samples. FEBS Lett 2009, 583:3966-3973.

4. Foss EJ, Radulovic D, Shaffer SA, Goodlett DR, Kruglyak L, Bedalov A: Genetic variation shapes protein networks mainly through non-transcriptional mechanisms. PLOS Bio/ 2011, 9:e1001144.

5. Ghazalpour A, Bennett B, Petyuk VA, Orozco L, Hagopian R, Mungrue IN, Farber CR, Sinsheimer J, Kang HM, Furlotte N, Park CC, Wen PZ, Brewer H, Weitz K, Camp DG, Pan C, Yordanova R, Neuhaus I, Tilford C, Siemers N, Gargalovic P, Eskin E, Kirchgessner T, Smith DJ, Smith RD, Lusis AJ: Comparative analysis of proteome and transcriptome variation in mouse. PLoS Genet 2011, 7:e1001393.

6. Vogel C, Marcotte EM: Insights into the regulation of protein abundance from proteomic and transcriptomic analyses. Nat ReV Genet 2012, 13:227-232

7. Maier T, Schmidt A, Güell M, Kühner S, Gavin AC, Aebersold R, Serrano L: Quantification of mRNA and protein and integration with protein turnover in a bacterium. Mol Syst Biol 2011, 7:511.

8. Schmidt A, Beck M, Malmström J, Lam H, Claassen M, Campbell D, Aebersold R: Absolute quantification of microbial proteomes at different states by directed mass spectrometry. Mol Syst Biol 2011, 7:510.

9. Greenbaum D, Colangelo C, Williams K, Gerstein M: Comparing protein abundance and mRNA expression levels on a genomic scale. Genome Biol 2003, 4:117.

10. Lee MV, Topper SE, Hubler SL, Hose J, Wenger CD, Coon JJ, Gasch AP. A dynamic model of proteome changes reveals new roles for transcript alteration in yeast. Mol Syst Biol 2011, 7:514.

11. Marquerat S, Schmidt A, Codlin S, Chen W, Aebersold R, Bähler J: Quantitative analysis of fission yeast transcriptomes and proteomes in proliferating and quiescent cells. Cell 2012, 151:671-683.

12. Lundberg E, Fagerberg L, Klevebring D, Matic I, Geiger T, Cox J, Algenäs C, Lundeberg J, Mann M, Uhlen M: Defining the transcriptome and proteome in three functionally different human cell lines. Mo/ Syst Biol 2010, 6:450

13. Nagaraj N, Wisniewski JR, Geiger T, Cox J, Kircher M, Kelso J, Pääbo S, Mann M: Deep proteome and transcriptome mapping of a human cancer cell line. Mol Syst Biol 2011, 7:548.

14. Tian Q, Stepaniants SB, Mao M, Weng L, Feetham MC, Doyle MJ, Yi EC, Dai H, Thorsson V, Eng J, Goodlett D, Berger JP, Gunter B, Linseley PS, Stoughton RB, Aebersold R, Collins SJ, Hanlon WA, Hood LE: Integrated genomic and proteomic analyses of gene expression in mammalian cells. Mol Cell Proteomics 2004, 3:960-969.

15. Graumann J, Hubner NC, Kim JB, Ko K, Moser M, Kumar C, Cox J, Schöler H, Mann M: Stable isotope labeling by amino acids in cell culture (SILAC) and proteome quantitation of mouse embryonic stem cells to a depth of 5,111 proteins. Mol Cell Proteomics 2008, 7:672-683.

16. Vogel C, de Sousa Abreu R, Ko D, Le SY, Shapiro BA, Burns SC, Sandhu D, Boutz DR, Marcotte EM, Penalva LO: Sequence signatures and mRNA concentration can explain two-thirds of protein abundance variation in a human cell line. Mol Syst Biol 2010, 6:400.

17. Schwanhäusser B, Busse D, Li N, Dittmar G, Schuchhardt J, Wolf J, Chen W, Selbach M: Global quantification of mammalian gene expression control. Nature 2011, 473:337-342.

18. Chia W, Somers WG, Wang H: Drosophila neuroblast asymmetric divisions: cell cycle regulators, asymmetric protein localization, and tumorigenesis. J Cell Bio/ 2008, 180:267-272

19. Doe CQ: Neural stem cells: balancing self-renewal with differentiation. Development 2008, 135:1575-1587.

20. Homem CCF, Knoblich JA: Drosophila neuroblasts: a model for stem cell biology. Development 2012, 139:4297-4310.

21. Arama E, Dickman D, Kimchie Z, Shearn A, Lev Z: Mutations in the beta-propeller domain of the Drosophila brain tumor (brat) protein induce neoplasm in the larval brain. Oncogene 2000, 19:3706-3716.

22. Loop T, Leemans R, Stiefel U, Hermida L, Egger B, Xie F, Primig M, Certa U, Fischbach KF, Reichert $\mathrm{H}$, Hirth F: Transcriptional signature of an adult brain tumor in Drosophila. BMC Genomics 2004, 5:24.

23. Betschinger J, Mechtler K, Knoblich JA: Asymmetric segregation of the tumor suppressor brat regulates self-renewal in Drosophila neural stem cells. Cell 2006, 124:1241-1253. 
24. Caussinus E, Gonzalez C: Induction of tumor growth by altered stem-cell asymmetric division in Drosophila melanogaster. Nat Genet 2005, 37:1125-1129.

25. Ross PL, Huang YN, Marchese JN, Williamson B, Parker K, Hattan S, Khainovski N, Pillai S, Dey S, Daniels S, Purkayastha S, Juhasz P, Martin S, Bartlet-Jones M, He F, Jacobson A, Pappin DJ: Multiplexed protein quantitation in Saccharomyces cerevisiae using amine-reactive isobaric tagging reagents. Mol Cell Proteomics 2004, 3:1154-1169.

26. Hebenstreit D, Fang M, Gu M, Charoensawan V, van Oudenaarden, A, Teichmann SA: RNA sequencing reveals two major classes of gene expression levels in metazoan cells. Mol Syst Biol 2011, 7:497.

27. Kanehisa M, Goto S, Furumichi M, Tanabe M, Hirakawa M: KEGG for representation and analysis of molecular networks involving diseases and drugs. Nucleic Acids Res 2010, 38:D355-D360.

28. Köcher T, Pichler P, Schutzbier M, Stingl C, Kaul A, Teucher N, Hasenfuss G, Penninger $J M$, Mechtler $K$ : High precision quantitative proteomics using ITRAQ on an LTQ Orbitrap: a new mass spectrometric method combining the benefits of all. J Proteome Res 2009, 8:4743-4752.

29. Lange V, Picotti $P$, Domon B, Aebersold R: Selected reaction monitoring for quantitative proteomics: a tutorial. Mol Syst Biol 2008, 4:222.

30. Ow SY, Salim M, Noirel J, Evans C, Rehman I, Wright PC, iTRAQ underestimation in simple and complex mixtures: iTRAQ underestimation in simple and complex mixtures: 'the good, the bad and the ugly'. J Proteome Res 2009, 8:5347-5355.

31. Evans C, Noirel J, Ow SY, Salim M, Pereira-Medrano AG, Couto N, Pandhal J, Smith D, Pham TK, Karunakaran E, Zou X, Biggs CA, Wright PC: An insight into iTRAQ: where do we stand now? Anal Bioanal Chem 2012, 404:1011-1027

32. Sharp PM, Li WH: The codon Adaptation Index - a measure of directional synonymous codon usage bias, and its potential applications. Nucleic Acids Res 1987, 15:1281-1295.

33. Brunner E, Ahrens $\mathrm{CH}$, Mohanty S, Baetschmann H, Loevenich S, Potthast F, Deutsch EW, Panse C, de Lichtenberg U, Rinner O, Lee H, Pedrioli PGA, Malmstrom J, Koehler K, Schrimpf S, Krijgsveld J, Kregenow F, Heck AJR, Hafen E, Schlapbach R, Aebersold R: A high-quality catalog of the Drosophila melanogaster proteome. Nat Biotechnol 2007 25:576-583.

34. Castellanos E, Dominguez P, Gonzalez C: Centrosome dysfunction in Drosophila neural stem cells causes tumors that are not due to genome instability. Curr Biol 2008, 18:1209-1214.

35. Gateff E: Malignant neoplasms of genetic origin in Drosophila melanogaster. Science 1978, 200:1448-1459.

36. Bello $B$, Reichert $H$, Hirth $F$ : The brain tumor gene negatively regulates neural progenitor cell proliferation in the larval central brain of Drosophila. Development 2006, 133:2639-2648.

37. Sandberg R, Neilson JR, Sarma A, Sharp PA, Burge CB: Proliferating cells express mRNAs with shortened 3' untranslated regions and fewer microRNA target sites. Science 2008, 320:1643-1647.

38. Mayr C, Bartel DP: Widespread shortening of 3' UTRs by alternative cleavage and polyadenylation activates oncogenes in cancer cells. Cell 2009, 138:673-684

39. Guruharsha KG, Rual JF, Zhai B, Mintseris J, Vaidya P, Vaidya N, Beekman C, Wong C, Rhee DY, Cenaj O, McKillip E, Shah S, Stapleton M, Wan KH, Yu C, Parsa B, Carlson JW, Chen X, Kapadia B, Vijayraghavan K, Gygi SP, Celniker SE, Obar RA, Artavanis-Tsakonas S: A protein complex network of Drosophila melanogaster. Cell 2011, 147:690-703.

40. Babu M, Vlasblom J, Pu S, Guo X, Graham C, Bean BDM, Burston HE, Vizeacoumar FJ, Snider J, Phanse S, Fong V, Tam YYC, Davey M, Hnatshak O, Bajaj N, Chandran S, Punna T, Christopolous C, Wong V, Yu A, Zhong G, Li J, Stagljar I, Conibear E, Wodak SJ, Emili A, Greenblatt JF: Interaction landscape of membrane-protein complexes in Saccharomyces cerevisiae. Nature 2012, 489:585-589.

41. Havugimana PC, Hart GT, Nepusz T, Yang H, Turinsky AL, Li Z, Wang PI, Boutz DR, Fong V, Phanse S, Babu M, Craig SA, Hu P, Wan C, Vlasblom J, Dar VuN, Bezginov A, Clark GW, Wu GC, Wodak SJ, Tillier ERM, Paccanaro A, Marcotte EM, Emili A: A census of human soluble protein complexes. Cell 2012, 150:1068-1081.

42. Ge H, Liu Z, Church GM, Vidal M: Correlation between transcriptome and interactome mapping data from Saccharomyces cerevisiae. Nat Genet 2001, 29:482-486.
43. Grigoriev A: A relationship between gene expression and protein interactions on the proteome scale: analysis of the bacteriophage T7 and the yeast Saccharomyces cerevisiae. Nucleic Acids Res 2001, 29:3513-3519.

44. Jansen R, Greenbaum D, Gerstein M: Relating whole-genome expression data with protein-protein interactions. Genome Res 2002, 12:37-46

45. Ori A, Banterle N, Iskar M, Andrés-Pons A, Escher C, Khanh Bui H, Sparks L, Solis-Mezarino V, Rinner O, Bork P, Lemke EA, Beck M: Cell type-specific nuclear pores: a case in point for context-dependent stoichiometry of molecular machines. Mol Syst Biol 2013, 9:648.

46. Cline MS, Smoot M, Cerami E, Kuchinsky A, Landys N, Workman C, Christmas R, Avila-Campilo I, Creech M, Gross B, Hanspers K, Isserlin R, Kelley R, Killcoyne S, Lotia S, Maere S, Morris J, Ono K, Pavlovic V, Pico AR, Vailaya A, Wang PL, Adler A, Conklin BR, Hood L, Kuiper M, Sander C, Schmulevich I, Schwikowski B, Warner GJ, et al.: Integration of biological networks and gene expression data using Cytoscape. Nat Protoc 2007, 2:2366-2382.

47. Hinnebusch AG: IF3: a versatile scaffold for translation initiation complexes. Trends Biochem Sci 2006, 31:553-562.

48. Zhang L, Pan X, Hershey JWB: Individual overexpression of five subunits of human translation initiation factor elF3 promotes malignant transformation of immortal fibroblast cells. J Biol Chem 2007, 282:5790-5800.

49. Hsu PP, Sabatini DM: Cancer cell metabolism: Warburg and beyond. Cell 2008, 134:703-707.

50. Ferreira LMR: Cancer metabolism: the Warburg effect today. Exp Mol Pathol 2010, 89:372-380.

51. Shim H, Dolde C, Lewis BC, Wu CS, Dang G, Jungmann RA, Dalla-Favera R, Dang CV: c-Myc transactivation of LDH-A: implications for tumor metabolism and growth. Proc Natl Acad Sci USA 1997, 94:6658-6663.

52. Fantin VR, St-Pierre J, Leder P: Attenuation of LDH-A expression uncovers a link between glycolysis, mitochondrial physiology, and tumor maintenance. Cancer Cell 2006, 9:425-434

53. Bochman ML, Schwacha A: The Mcm complex: unwinding the mechanism of a replicative helicase. Microbiol Mol Biol Rev 2009, 73:652-683.

54. Houseley J, LaCava J, Tollervey D: RNA-quality control by the exosome. Nat Rev Mol Cell Biol 2006, 7:529-539.

55. Andrulis ED, Werner J, Nazarian A, Erdjument-Bromage H, Tempst P, Lis JT: The RNA processing exosome is linked to elongating RNA polymerase II in Drosophila. Nature 2002, 420:837-841.

56. Spiess C, Meyer AS, Reissmann S, Frydman J: Mechanism of the eukaryotic chaperonin: protein folding in the chamber of secrets. Trends Cell Biol 2004, 14:598-604.

57. Hartl FU, Bracher A, Hayer-Hartl M: Molecular chaperones in protein folding and proteostasis. Nature 2011, 475:324-332.

58. Neumüller RA, Richter C, Fischer A, Novatchkova M, Neumüller KG, Knoblich JA: Genome-wide analysis of self-renewal in Drosophila neural stem cells by transgenic RNAi. Cell Stem Cell 2011, 8:580-593.

59. Mortazavi A, Williams BA, McCue K, Schaeffer L, Wold B: Mapping and quantifying mammalian transcriptomes by RNA-Seq. Nat Methods 2008, 5:621-628.

60. Langmead B, Trapnell C, Pop M, Salzberg SL: Ultrafast and memory-efficient alignment of short DNA sequences to the human genome. Genome Biol 2009, 10:R25

61. Trapnell C, Pachter $L$, Salzberg SL: TopHat: discovering splice junctions with RNA-Seq. Bioinformatics 2009, 25:1105-1111.

62. Trapnell C, Williams BA, Pertea G, Mortazavi A, Kwan G, van Baren, M J, Salzberg SL, Wold BJ, Pachter L: Transcript assembly and quantification by RNA-Seq reveals unannotated transcripts and isoform switching during cell differentiation. Nat Biotechnol 2010, 28:511-515.

63. Roberts A, Trapnell C, Donaghey J, Rinn JL, Pachter L: Improving RNA-Seq expression estimates by correcting for fragment bias. Genome Biol 2011, 12:R22.

64. ProteomeXchange consortium. [http://proteomecentral. proteomexchange.org]

65. Vizcaíno JA, Côté RG, Csordas A, Dianes JA, Fabregat A, Foster JM, Griss J, Alpi E, Birim M, Contell J, O'Kelly G, Schoenegger A, Ovelleiro D, Pérez-Riverol Y, Reisinger F, Ríos D, Wang R, Hermjakob H: The 
PRoteomics IDEntifications (PRIDE) database and associated tools: status in 2013. Nucleic Acids Res 2013, 41:D1063-D1069.

66. McQuilton P, St-Pierre SE, Thurmond J, Consortium F: FlyBase 101 - the basics of navigating FlyBase. Nucleic Acids Res 2012, 40:D706-D714.

67. Elias JE, Gygi SP: Target-decoy search strategy for increased confidence in large-scale protein identifications by mass spectrometry. Nat Methods 2007, 4:207-214.

68. Perkins DN, Pappin DJC, Creasy DM, Cottrell JS: Probability-based protein identification by searching sequence databases using mass spectrometry data. Electrophoresis 1999, 20:3551-3567.

69. Eng JK, McCormack AL, Yates JR: An approach to correlate tandem mass spectral data of peptides with amino acid sequences in a protein database. J Am Soc Mass Spect 1994, 5:976-989.

70. Craig R, Beavis RC: TANDEM: matching proteins with tandem mass spectra. Bioinformatics 2004, 20:1466-1467.

71. Sadygov RG, Good DM, Swaney DL, Coon JJ: A new probabilistic database search algorithm for ETD spectra. J Proteome Res 2009, 8:3198-3205

72. Qeli $\mathrm{E}$, Ahrens $\mathrm{CH}$ : PeptideClassifier for protein inference and targeted quantitative proteomics. Nat Biotechnol 2010, 28:647-650

73. Jones AR, Siepen JA, Hubbard SJ, Paton NW: Improving sensitivity in proteome studies by analysis of false discovery rates for multiple search engines. Proteomics 2009, 9:1220-1229.

74. Karp NA, Huber W, Sadowski PG, Charles PD, Hester SV, Lilley KS: Addressing accuracy and precision issues in ITRAQ quantitation. Mol Cell Proteomics 2010, 9:1885-1897.

75. Käll L, Storey JD, MacCoss MJ, Noble WS: Assigning significance to peptides identified by tandem mass spectrometry using decoy databases. J Proteome Res 2008, 7:29-34

76. PeptideAtlas project. [http://www.peptideatlas.org]

77. R Core Team: A Language and Environment for Statistical Computing. Vienna: R Foundation for Statistical Computing; 2012

78. Rice P, Longden I, Bleasby A: EMBOSS: the European Molecular Biology Open Software Suite. Trends Genet 2000, 16:276-277.

79. Kyte J, Doolittle RF: A simple method for displaying the hydropathic character of a protein. $J$ Mol Biol 1982, 157:105-132.

80. Azzalini A: R package sn: The skew-normal and skew-t distributions (version 0.4-18). Italia: Università di Padova; 2013.

doi:10.1186/gb-2013-14-11-r133

Cite this article as: Jüschke et al:: Transcriptome and proteome quantification of a tumor model provides novel insights into post-transcriptional gene regulation. Genome Biology 2013 14:r133.

Submit your next manuscript to BioMed Central and take full advantage of:

- Convenient online submission

- Thorough peer review

- No space constraints or color figure charges

- Immediate publication on acceptance

- Inclusion in PubMed, CAS, Scopus and Google Scholar

- Research which is freely available for redistribution

Submit your manuscript at www.biomedcentral.com/submit
C) Biomed Central 